\title{
Effect of short-course antibiotics on the antimicrobial resistance profile of colonizing gut bacteria in the ICU
}

\section{Christian Munck}

Columbia University

\section{Ravi Sheth}

Columbia University

\section{Edward Cuaresma}

Columbia University

\section{Stephania Stump}

Columbia University

Jessica Weidler

Columbia University

Philip Zachariah

Columbia University

\section{David Chong}

Columbia University

Anne-Catrin Uhlemann

Columbia University

Julian Abrams

Columbia University

Harris Wang

Columbia University

Daniel E Freedberg ( $\sim$ def2004@cumc.columbia.edu )

Columbia University Medical Center https://orcid.org/0000-0003-2023-2899

\section{Research}

Keywords: antimicrobial resistance, antibiotics, sepsis, colonization, healthcare-associated infection

Posted Date: November 13th, 2019

DOI: https://doi.org/10.21203/rs.2.17232/v1 
License: (c) (i) This work is licensed under a Creative Commons Attribution 4.0 International License. Read Full License 
1 Effect of short-course antibiotics on the antimicrobial resistance profile of colonizing gut

2 bacteria in the ICU

3

4 Christian Munck, PhD, ${ }^{1}$ Ravi U. Sheth, $\mathrm{PhD},{ }^{1}$ Edward Cuaresma, MD ${ }^{2}$ Jessica Weidler, BA, ${ }^{3}$

5 Stephania Stump, BA, ${ }^{3}$ Philip Zachariah, MD, MS, ${ }^{4}$ David H. Chong, MD, ${ }^{5}$ Anne-Catrin

6 Uhlemann, MD, PhD,${ }^{3}$ Julian A. Abrams, MD, MS, ${ }^{6}$ Harris H. Wang, PhD, ${ }^{1}$ Daniel E. Freedberg,

$7 \mathrm{MD}, \mathrm{MS}^{6}$

8

$9 \quad{ }^{1}$ Department of System Biology, Columbia University Irving Medical Center

$10 \quad{ }^{2}$ Department of Medicine, Columbia University Irving Medical Center

$11 \quad{ }^{3}$ Division of Infectious Diseases, Columbia University Irving Medical Center

$12{ }^{4}$ Division of Pediatric Infectious Diseases, Columbia University Irving Medical Center

$13{ }^{5}$ Division of Pulmonary, Allergy, and Critical Care Medicine, Columbia University Irving Medical

14 Center

$15{ }^{6}$ Division of Digestive and Liver Diseases, Columbia University Irving Medical Center

16

17 Running title: Effect of short-course antibiotics on resistance

18

19 Correspondence: Daniel E. Freedberg, Division of Digestive and Liver Diseases, Columbia 20 University Medical Center, 630 West $168^{\text {th }}$ Street, P\&S 3-401, New York, NY 10032; Tel (212)

21 305-1021, Fax (212) 305-5576, E-mail: def2004@cumc.columbia.edu or Christian Munck,

22 Department of Systems Biology, Columbia University Medical Center, 3960 Broadway, New

23 York, NY 10032; Tel (212-305-1697, E-mail: cm3297@ cumc.columbia.edu. 
1 Key words: antimicrobial resistance, antibiotics, sepsis, colonization, healthcare-associated

2 infection

3

4

5

6

7 
3 Background: The need for early antibiotics in the intensive care unit (ICU) is often balanced

4 against the goal of antibiotic stewardship. We sought to determine how short-course antibiotics in 5 the ICU affect the antimicrobial resistance phenotype and genotype of colonizing gut bacteria.

7 Results: Deep rectal swabs were performed on 48 adults at the time of ICU admission and 72

8 hours later, including patients who did and did not receive antibiotics. To determine resistance 9 phenotype, rectal swabs were cultured for MRSA and VRE; in addition, Gram-negative isolates 10 were cultured against relevant antibiotics. To determine resistance genotype, quantitative PCR 11 was performed for 87 established resistance genes. Within-individual changes in antimicrobial 12 resistance were calculated based on culture and qPCR results and correlated with exposure to 13 relevant antibiotics (e.g., $\beta$-lactam exposure and changes in $\beta$-lactam resistance). Of 48 ICU 14 patients, $41(85 \%)$ received antibiotics. Overall, there was no increase in the antimicrobial 15 resistance profile of colonizing gut bacteria during the 72 hour study period. There was also no 16 increase in antimicrobial resistance after stratification by receipt of antibiotics (i.e., no increase in $17 \beta$-lactam, vancomycin, or macrolide resistance regardless of whether patients received those same 18 antibiotics). This was true for both culture and PCR. Antimicrobial resistance pattern at ICU 19 admission strongly predicted resistance pattern after 72 hours.

21 Conclusions. Short-course ICU antibiotics made little difference in the antimicrobial resistance 22 pattern of colonizing gut bacteria over 72 hours in the ICU. This provides improved understanding 
1 of the dynamics of antimicrobial resistance in the ICU and some reassurance that short-course

2 antibiotics may not adversely impact the stewardship goal of reducing antimicrobial resistance.

3

4

5

6 
INTRODUCTION

3

4

\section{5}

6

0

5

6

21

Empiric antibiotics are the main pillar of sepsis treatment in the intensive care unit (ICU). There is a $10-42 \%$ absolute increase in sepsis mortality when initial empiric antibiotics fail to appropriately treat infecting organisms [1-3]. Clinical guidelines recommend that broad-spectrum, empiric antibiotics be initiated as part of a treatment bundle within 1 hour of presentation with sepsis [4], and studies suggest that outcomes may improve when antibiotics are given as quickly as possible [5].

Balanced against the imperative for early, broad-spectrum antibiotics is the mandate for antibiotic stewardship. Using fewer or more narrow spectrum antibiotics avoids drug-drug interactions, avoids antibiotic side effects, and furthers the stewardship goal of minimizing the emergence of antimicrobial resistance [6].

Long courses of antibiotics in the ICU are associated with increased gastrointestinal antimicrobial resistance [7]. The impact of short-course antibiotics on antimicrobial resistance in the ICU is less certain. Large structural microbiome changes can be seen within 72 hours after oral antibiotic intake in healthy, antibiotic-naïve volunteers [8]. Whether antimicrobial resistance can emerge so quickly following intravenous antibiotics in the ICU is not known. admission and 72 hours later, and compared changes in gastrointestinal antimicrobial resistance in 
1 those who did or did not receive antibiotics among different classes. The goal of the study was to

2 determine if short-term antibiotics adversely impact gastrointestinal antimicrobial resistance in the 3 ICU.

4

5 RESULTS

6

7 Population

A total of 48 critically ill patients were included in the study and swabbed at the time of

9 ICU admission and 72 hours later (Table 1). This 72-hour window was selected because antibiotic

10 discontinuation in the ICU is often first considered after 72 hours of antibiotic treatment. Median

11 SOFA score was 16 (IQR, 10-18) at ICU admission and 17 (IQR, 15-19) after 72 hours

12 (Supplemental Table 2). Raw data for the study is given in Data Supplement 1 (phenotype) and

13 Data Supplement 2 (genotype).

14

15

16

17

18

19

20

21

22

23

\section{Receipt of antibiotics}

Patients were eligible for inclusion in the study if they did or did not receive antibiotics. In sum, 41/48 (85\%) of patients in the study received antibiotics and 37/48 (77\%) received broadspectrum antibiotics, most often a $3^{\text {rd }}$ generation cephalosporin or an extended spectrum penicillin with a $\beta$-lactamase inhibitor. Figure 1A shows the antibiotics received by class, and Figure 1B shows pairwise combinations of antibiotics. Almost all antibiotics were intravenous. Of 91 antibiotics dosed to the 48 patients, 93\% were given intravenously (1 patient received oral azithromycin, 1 received oral vancomycin, and 4 received oral rifaximin). 


\section{Overall changes in antimicrobial resistance phenotype and genotype}

0

First, antimicrobial resistance phenotype was examined by culturing rectal swabs and performing susceptibility testing. No differences were evident comparing summary data for resistance phenotype at ICU admission versus 72 hours later for MRSA (chi-squared $p=0.59$ ), VRE $(p=0.61)$, or Gram-negative bacteria showing $\beta$-lactam resistance $(p=0.27$ (Supplemental Figure 1A). Summing all antimicrobial resistance phenotype categories, there were no differences in rates of antimicrobial resistance for Gram-negative bacteria comparing ICU admission (nonsusceptibility for 88/960 antibiotics tested, 9.1\%) versus 72 hours later (non-susceptibility for 107/960 antibiotics tested, 11.1\%) (chi-squared $\mathrm{p}=0.17)$.

Next, antimicrobial resistance genotype was examined by performing qPCR across 87 common antibiotic resistance genes from the rectal swabs. No differences were evident comparing summary genotype data from ICU admission versus 72 hours later for genes conferring resistance to $\beta$-lactams (chi-squared $\mathrm{p}=0.27)$, vancomycin $(\mathrm{p}=1.0)$, macrolides $(\mathrm{p}=0.93)$, or fluoroquinolones $(\mathrm{p}=0.74)$ (Supplemental Figure 1B). Summing all resistance genes, there were no differences in rates of antimicrobial resistance comparing ICU admission (positive qPCR for 392 of 4,176 genes, 9\%) versus 72 hours later (positive qPCR for 421 of 4,176 genes, 10\%) (chisquared $\mathrm{p}=0.30)$.

\section{Effect of antibiotics on antimicrobial resistance phenotype}

For patients that did not carry individual resistant bacteria at admission, the carriage rate after 72 hours for those that received relevant antibiotics was compared to the carriage rate for those that did not receive relevant antibiotics (e.g., comparing $\beta$-lactam non-susceptibility in 
1 culture based on receipt of $\beta$-lactam antibiotics). Antibiotics had no significant association with

2 resistance phenotype (Figure 2). Presence of Gram-negative bacteria showing resistance to at least

3 one $\beta$-lactam antibiotic after 72 hours was seen in $8 / 24(33 \%)$ of patients who received $\beta$-lactam

4 antibiotics and in 2/10 (20\%) of patients who did not (Fisher's p = 0.68). VRE was present in

$5 \quad 1 / 13(8 \%)$ patients who received vancomycin and in $2 / 27(7 \%)$ of patients who did not $(\mathrm{p}=1.0)$.

6 MRSA was present in 5/31 (16\%) patients who received $\beta$-lactams and in $0 / 10(0 \%)$ of patients

$7 \quad$ who did not $(\mathrm{p}=0.31)$.

8

9 Effect of antibiotics on antimicrobial resistance genotype

Change in antimicrobial resistance gene abundance was tested after stratifying by receipt

11 of antibiotic class (Figure 3). This was first done with change in genotype classified as a 12 continuous variable based on within-individual change in $\mathrm{C}_{\mathrm{T}}$ values (ICU admission $\mathrm{C}_{\mathrm{T}}$ minus 72 -

13 hour $\mathrm{C}_{\mathrm{T}}$ ). There was no association between the changes within relevant resistance genes after 72

14 hours and receipt of any of the 3 most common antibiotic categories ( $\beta$-lactams, vancomycin, or

15 macrolides). There was also no overall difference in the within-individual change in $\mathrm{C}_{\mathrm{T}}$ values

16 comparing combined antibiotic gene categories ( $\mathrm{t}$-test $\mathrm{p}=0.49$ for $\beta$-lactams, $\mathrm{p}=0.28$ for

17 vancomycin, and $\mathrm{p}=0.19$ for macrolides) (Figure 3). This analysis was then repeated classifying

18 within-individual change in genotype categorically (i.e., present versus absent). Again, there was

19 no association between receipt of antibiotics and changes within relevant antibiotic resistance 20 genotypes.

21

22 ICU admission antimicrobial resistance pattern as a predictor of resistance phenotype and 23 genotype after 72 hours 

resistance after 72 hours for both phenotype and genotype. For 7 of the 9 antimicrobial resistance categories tested, presence of the resistant phenotype (i.e., non-susceptibility in culture) at the time

4 of ICU admission was significantly associated with non-susceptibility 72 hours later (Figure 4A).

5 For 14 of the 26 genes tested, presence of the resistance genotype (i.e., positive qPCR) at the time 6 of ICU admission was significantly associated with positive qPCR 72 hours later (Figure 4B).

\section{DISCUSSION}

In this study of 48 critically ill patients, there was no association between receipt of short-

12 course antibiotics and change in antimicrobial resistance phenotype or genotype during the 72

13 hours following ICU admission. Initial patient-level ICU antimicrobial resistance pattern was the 14 best predictor of antimicrobial resistance after 72 hours, and significant interim changes were rare. 15 Results were consistent when antimicrobial resistance genotype was assayed instead of phenotype.

This study addressed the question of short-term resistance dynamics within the gut 18 microbiome of ICU patients. Clinically, the imperative for early broad-spectrum antibiotics in the 19 ICU is balanced against the desire for antibiotic stewardship. Decisions regarding use of 20 antibiotics for sepsis are usually made before diagnostic microbiology results are available $[9,10]$.

21 Can intensivists reasonably give and continue empiric broad-spectrum antibiotics for 72 hours 22 without excessive concern that they are promoting antimicrobial resistance? Our results are 23 reassuring, but must be interpreted with caution. Prior studies clearly establish that antimicrobial 
1 resistance arises within colonizing gut bacteria during long-term antibiotic treatment in the ICU.

2 The question is not if resistance develops but when. The 72-hour treatment window in this study

3 parallels the 3-day interval after which antibiotic discontinuation is sometimes considered in the

4 ICU $[4,11]$. The results suggest that 72 hours is not long enough for the development of new

5 gastrointestinal antimicrobial resistance within a given individual. Longer antibiotic treatment

6 window periods would almost certainly have led to different study results.

These findings regarding the dynamics of resistance contrast sharply with in vitro studies.

9 When bacterial isolates are exposed to a selective pressure in culture, mutations that confer 10 antimicrobial resistance are rapidly selected $[12,13]$. Compared to in vitro systems, the gut 11 microbiome of ICU patients has many competing selection pressures. This dense network of 12 interactions may delay selection for antimicrobial resistance genes [14]. In ICU patients, collapse 13 of the pre-existing gut microbiome and emergence of a pathobiome enriched in resistance may 14 require 11-14 days of antibiotic treatment $[15,16]$. Other studies suggest that resistance does 15 emerge, but does so slowly. In allogeneic stem cell transplant patients, emergence of new 16 resistance within multiple VRE clones was seen after 7 days of selective antibiotics, with most 17 new resistance observed after 3 weeks or more [17]. In an infant treated with multiple antibiotics, 182 months were required before antimicrobial resistance emerged within specific bacterial lineages $19[18,19]$. In a similar study, novel plasmid-mediated ampicillin resistance was acquired after 162032 days in the absence of antibiotic treatment [20]. The implication is that resistance within 21 complex human systems such as the gut arises over weeks instead of days [7]. 
Multiple factors probably contributed to the relatively modest changes observed in

2

4

0

antimicrobial resistance. The antibiotics received were $93 \%$ intravenous. Intravenous antibiotics do penetrate into the gut [21], but luminal concentrations and pharmacodynamics may matter [22]. In ICU patients, Ravi et al. found that meropenem, but not piperacillin-tazobactam, was associated with loss of community structure as evidenced by loss of diversity [23]. Another possibility is that patients were already too enriched in antimicrobial resistance at the time of ICU admission because of past antibiotic exposures to detect a meaningful change in resistance over 72 hours. Prior studies support such a conclusion. Willmann et al. found surprisingly little gains in fluoroquinolone resistance during prophylaxis of neutropenic patients, perhaps because of past exposures [24].

11

This study has limitations. It did not seek to correlate antimicrobial resistance with specific bacterial lineages and cannot state whether "new" antimicrobial resistance was acquired from the environment, from horizontal gene transfer, or vertically within bacterial lineages. Such correlations are technically challenging [25]. Rather, a standard clinical culture-based approach was used to identify resistance within the primarily Gram-negative bacteria that cause most serious ICU infections [26]. Then quantitative PCR was added to determine resistance genotype. Alternative methodologies could have been used for genotyping [27], but qPCR was selected for ease of performance and high sensitivity [28]. Last, the study was relatively small and not powered to detect a very weak relationship between antibiotics and antimicrobial resistance. Given the large historical benefits attributed to antibiotics, a modest effect on antimicrobial resistance is unlikely to significantly alter the clinical risk-benefit calculation. 

resistance within colonizing gut bacteria during the initial 72 hours in the ICU. This was the case

3 for resistance phenotype based on culture and resistance genotype based on qPCR. Antimicrobial

4 resistance was relatively stable between ICU admission and the 72 hour mark. This result may

5 provide some reassurance that short-course antibiotics given at ICU admission do not necessarily

6 have an adverse effect on individuals' antimicrobial resistance.

\section{METHODS}

\section{Population}

A random subset of 48 patients was selected from a larger, previously described

12 prospective cohort parent study of over 200 ICU patients $[29,30]$. The sample size of 48 patients

13 was chosen as a compromise between statistical power and the substantial cost associated with the

14 assays. Adults $\geq 18$ years old were eligible for the study if they were emergently admitted to the

15 ICU from 2017 to 2019 at our institution, and if rectal swabs could be obtained within 4 hours of 16 ICU admission and again 72 hours later (+/- 4 hours). This 72-hour timeframe was selected

17 because it represents a common minimum period after which antibiotic discontinuation might be 18 considered.[4] All patients in the study had single rooms (no roommates) and gown and glove 19 contact precautions were used for the duration of the study for patients with known MRSA, VRE, 20 C. difficile, or ESBL Gram-negative colonization. Informed consent was obtained from all 21 subjects or from appropriate surrogates when subjects lacked capacity. The study was approved 22 by the institutional review board of Columbia University. 


\section{Study assessments}

2 9 immediately preceding the 72 hour mark (corresponding to the second study assessment). ICU

At each study assessment (ICU admission and 72 hours later), samples were taken and clinical information was gathered. Two duplicate deep rectal flocked nylon swabs [31] were collected with the patient in the lateral decubitus position, with fecal staining of swabs used to verify adequate sampling. Demographic information, laboratory data, and data related to interventions performed in the ICU between study visits was extracted from the electronic medical record. For laboratory data, test results were used from the first venous blood draw in the ICU (corresponding to the first study assessment) and from a venous blood draw either at or interventions were recorded including antibiotics, proton pump inhibitors, mechanical ventilation, hemodialysis, and enteral feeding. Clinical and laboratory data were used to estimate acute severity of illness according to the Sequential Organ Failure Assessment (SOFA) score as recommended by Sepsis-3 [32].

\section{Receipt of antibiotics}

The study enrolled patients who did and did not receive antibiotics during the initial 72 hours in the ICU. Use of antibiotics, and which antibiotics were used, was determined by treating ICU teams based on clinical need, without reference to the study. Receipt of antibiotics was classified categorically, without respect to number of doses or route of administration, based on whether antibiotics were received in the ICU between the initial admission rectal swab and the 72hour rectal swab. For this study, antibiotics were considered broad-spectrum if they fell within the following class categories: $\beta$-lactam/ $\beta$-lactamase inhibitor combination antibiotics, carbapenems, cephalosporins, fluoroquinolones, and lincosamides. This definition covers 5 of the 
17 most commonly prescribed classes of antibiotics in U.S. hospitals [33]. The term $\beta$-lactams has

2 been used to describe ampicillin, $\beta$-lactam/ $\beta$-lactamase combination antibiotics, carbapenems, and 3 cephalosporins.

4

\section{Resistance phenotype}

$6 \quad$ Resistance phenotype was determined using selective and non-selective cultures. Rectal 7 swabs were inoculated into soy broth with $20 \%$ glycerol media at the bedside. After gentle mixing, 8 these swabs were plated on 3 media: (1) selective chromogenic media for VRE including $E$. 9 faecalis or E. faecium; (2) selective chromogenic media for MRSA; and (3) MacConkey II agar 10 for Gram-negative bacteria. All plates were incubated aerobically at $33-37^{\circ} \mathrm{C}$ and assessed after $11>24$ hours. VRE and MRSA were classified as present versus absent according to the

12 manufacturer's instructions. Resistance phenotype for Gram-negative isolates was determined 13 using the VITEK 2 system and AST-N010/020 cards with confirmatory testing as needed. Routine 14 Clinical and Laboratory Standards Institute (CLSI) cut-offs were used for non-susceptibility [34].

\section{Resistance genotype}

Resistance genotype was determined using a quantitative PCR kit that assesses 87 common

18 antibiotic resistance genes (complete list of genes in Supplemental Table 1, Qiagen Cat. No.

19 330261) [35]. To do this, DNA was extracted from the duplicate rectal swab and $250 \mathrm{ng}$ of 20 template meta-genomic DNA was added to each reaction on a 96-well RT-PCR plate and run 21 according to the manufacturer's protocol. Controls were used to detect the presence of bacterial 22 DNA, PCR inhibitors, and background. Adequate reactions were determined by $\mathrm{C}_{\mathrm{T}}$ values of $<$ 2329 for the pan-bacterial reference genes $16 S r R N A$, gyrA, recA, and $r p o B$ and positive PCR control 
1 cycle thresholds $\left(\mathrm{C}_{\mathrm{T}}\right)$ values of $<24$ [36]. Genotype was classified both as a continuous variable

2 based on $\mathrm{C}_{\mathrm{T}}$ value and also, per assay recommendations, as a categorical variable with $\mathrm{C}_{\mathrm{T}}$ values

3 of $\leq 34$ considered positive for the presence of a given gene.

4

$5 \quad$ Statistical approach

6 Summary data was compared using chi-squared tests or Fisher's test when cell counts were

$7<5$. For continuous summary data, t-tests or rank-sum tests were used when the data was not

8 normal in distribution. Chi-squared or Fisher's tests were used to compare resistance-related

9 outcomes that were classified categorically; patients who already showed resistance at ICU

10 admission were excluding from such testing, because they did not have the possibility of

11 developing new colonization. The final sample size of 48 patients gave $80 \%$ power to detect a

12 difference in paired means representing antimicrobial resistance genotype of 0.41 standard

13 deviations, with resistance genotype classified as a continuous variable based on $\mathrm{C}_{\mathrm{T}}$ value. All

14 testing was done two-sided at an alpha 0.05 level of significance using R.

16 LIST OF ABBREVIATONS

17 ICU: intensive care unit; IQR: interquartile range; MDR: multidrug resistant; MRSA: methicillin18 resistant Staphylococcus aureus; PCR: polymerase chain reaction; VRE: vancomycin-resistant 19 Enterococcus 
DECLARATIONS

2 Ethics approval and consent to participate: This study was approved by the institutional review

3 board of Columbia University with participation from subjects, or from surrogates when subjects

4 lacked capacity.

5 Consent for publication: Not applicable.

6 Availability of data and materials: Complete raw data for the study has been made available as

7 an on-line supplement at [hyperlink to come from journal].

8 Competing interests: The authors declare that they have no competing interests.

9 Funding: CM was supported in part by the Carlsberg Foundation, RUS was supported in part by

10 a Fannie and John Hertz Foundation Fellowship and by a NSF Graduate Research Fellowship

11 (DGE-1644869), ACU was supported in part by NIAID R01 AI116939, and JAA was supported

12 in part by NCI U54 CA163004, HHW was supported in part by NIAID R01AI132403, NIDDK

13 R01DK118044, and the Burroughs Welcome Fund PATH (1016691), and DEF was supported in

14 part by NIDDK K23 DK111847. ACU has received unrelated funding from Merck, GSK and

15 Allergan. None of the funding organizations had any direct involvement in the study.

16 Authors' contributions: DEF, CM, RS, and JAA contributed to the design and conception of the

17 study; EC, JW, SS, DHC, and ACU contributed to data acquisition; and DEF and CM conducted

18 statistical analyses. DEF and CM drafted the manuscript and all authors have interpreted the data

19 and provided critical revisions. All authors have read and approved the final manuscript.

20 Acknowledgements: Not applicable.

21 


\section{REFERENCES}

1. Paul M, Shani V, Muchtar E, Kariv G, Robenshtok E, Leibovici L: Systematic review and meta-analysis of the efficacy of appropriate empiric antibiotic therapy for sepsis. Antimicrob Agents Chemother 2010, 54(11):4851-4863.

2. Kumar A, Ellis P, Arabi Y, Roberts D, Light B, Parrillo JE, Dodek P, Wood G, Kumar A, Simon D et al: Initiation of inappropriate antimicrobial therapy results in a fivefold reduction of survival in human septic shock. Chest 2009, 136(5):1237-1248.

3. Ferrer R, Artigas A, Suarez D, Palencia E, Levy MM, Arenzana A, Perez XL, Sirvent JM, Edusepsis Study G: Effectiveness of treatments for severe sepsis: a prospective, multicenter, observational study. Am J Respir Crit Care Med 2009, 180(9):861-866.

4. Rhodes A, Evans LE, Alhazzani W, Levy MM, Antonelli M, Ferrer R, Kumar A, Sevransky JE, Sprung CL, Nunnally ME et al: Surviving Sepsis Campaign: International Guidelines for Management of Sepsis and Septic Shock: 2016. Crit Care Med 2017, 45(3):486-552.

5. Seymour CW, Gesten F, Prescott HC, Friedrich ME, Iwashyna TJ, Phillips GS, Lemeshow S, Osborn T, Terry KM, Levy MM: Time to Treatment and Mortality during Mandated Emergency Care for Sepsis. N Engl J Med 2017, 376(23):2235-2244.

6. Organisation for Economic Cooperation and Development. Stemming the superbug tide: just a few dollars more. 7 Nov 2018. Accessed on-line April 15, 2019 at https://doi.org/10.1787/9789264307599-en.

7. Filius PM, Gyssens IC, Kershof IM, Roovers PJ, Ott A, Vulto AG, Verbrugh HA, Endtz HP: Colonization and resistance dynamics of gram-negative bacteria in patients during and after hospitalization. Antimicrob Agents Chemother 2005, 49(7):2879-2886.

8. Dethlefsen L, Relman DA: Incomplete recovery and individualized responses of the human distal gut microbiota to repeated antibiotic perturbation. Proc Natl Acad Sci U S A 2011, 108 Suppl 1:4554-4561.

9. Whiles BB, Deis AS, Simpson SQ: Increased Time to Initial Antimicrobial Administration Is Associated With Progression to Septic Shock in Severe Sepsis Patients. Crit Care Med 2017, 45(4):623-629.

10. Liu VX, Fielding-Singh V, Greene JD, Baker JM, Iwashyna TJ, Bhattacharya J, Escobar GJ: The Timing of Early Antibiotics and Hospital Mortality in Sepsis. Am J Respir Crit Care Med 2017, 196(7):856-863.

11. Dellit TH, Owens RC, McGowan JE, Jr., Gerding DN, Weinstein RA, Burke JP, Huskins WC, Paterson DL, Fishman NO, Carpenter CF et al: Infectious Diseases Society of America and the Society for Healthcare Epidemiology of America guidelines for developing an institutional program to enhance antimicrobial stewardship. Clin Infect Dis 2007, 44(2):159-177.

12. Good BH, McDonald MJ, Barrick JE, Lenski RE, Desai MM: The dynamics of molecular evolution over 60,000 generations. Nature 2017, 551(7678):45-50.

13. Tenaillon O, Barrick JE, Ribeck N, Deatherage DE, Blanchard JL, Dasgupta A, Wu GC, Wielgoss S, Cruveiller S, Medigue $\mathrm{C}$ et al: Tempo and mode of genome evolution in a 50,000-generation experiment. Nature 2016, 536(7615):165-170.

14. Lescat M, Launay A, Ghalayini M, Magnan M, Glodt J, Pintard C, Dion S, Denamur E, Tenaillon O: Using long-term experimental evolution to uncover the patterns and 
determinants of molecular evolution of an Escherichia coli natural isolate in the streptomycin-treated mouse gut. Mol Ecol 2017, 26(7):1802-1817.

15. Alverdy JC, Krezalek MA: Collapse of the Microbiome, Emergence of the Pathobiome, and the Immunopathology of Sepsis. Crit Care Med 2017, 45(2):337-347.

16. Perez-Cobas AE, Gosalbes MJ, Friedrichs A, Knecht H, Artacho A, Eismann K, Otto W, Rojo D, Bargiela R, von Bergen $\mathrm{M}$ et al: Gut microbiota disturbance during antibiotic therapy: a multi-omic approach. Gut 2013, 62(11):1591-1601.

17. Dubin KA, Mathur D, McKenney PT, Taylor BP, Littmann ER, Peled JU, van den Brink MRM, Taur Y, Pamer EG, Xavier JB: Diversification and evolution of vancomycinresistant Enterococcus faecium during intestinal domination. Infect Immun 2019.

18. Porse A, Gumpert H, Kubicek-Sutherland JZ, Karami N, Adlerberth I, Wold AE, Andersson DI, Sommer MOA: Genome Dynamics of Escherichia coli during Antibiotic Treatment: Transfer, Loss, and Persistence of Genetic Elements In situ of the Infant Gut. Front Cell Infect Microbiol 2017, 7:126.

19. Karami N, Martner A, Enne VI, Swerkersson S, Adlerberth I, Wold AE: Transfer of an ampicillin resistance gene between two Escherichia coli strains in the bowel microbiota of an infant treated with antibiotics. J Antimicrob Chemother 2007, 60(5):1142-1145.

20. Gumpert H, Kubicek-Sutherland JZ, Porse A, Karami N, Munck C, Linkevicius M, Adlerberth I, Wold AE, Andersson DI, Sommer MOA: Transfer and Persistence of a MultiDrug Resistance Plasmid in situ of the Infant Gut Microbiota in the Absence of Antibiotic Treatment. Front Microbiol 2017, 8:1852.

21. Urtasun RC, Rabin HR, Partington J: Human pharmacokinetics and toxicity of high-dose metronidazole administered orally and intravenously. Surgery 1983, 93(1 Pt 2):145-148.

22. Tulstrup MV, Christensen EG, Carvalho V, Linninge C, Ahrne S, Hojberg O, Licht TR, Bahl MI: Antibiotic Treatment Affects Intestinal Permeability and Gut Microbial Composition in Wistar Rats Dependent on Antibiotic Class. PLoS One 2015, 10(12):e0144854.

23. Ravi A, Halstead FD, Bamford A, Casey A, Thomson NM, van Schaik W, Snelson C, Goulden R, Foster-Nyarko E, Savva GM et al: Loss of microbial diversity and pathogen domination of the gut microbiota in critically ill patients. Microb Genom 2019, 5(9).

24. Willmann M, Vehreschild M, Biehl LM, Vogel W, Dorfel D, Hamprecht A, Seifert H, Autenrieth IB, Peter S: Distinct impact of antibiotics on the gut microbiome and resistome: a longitudinal multicenter cohort study. BMC Biol 2019, 17(1):76.

25. Ferreiro A, Crook N, Gasparrini AJ, Dantas G: Multiscale Evolutionary Dynamics of HostAssociated Microbiomes. Cell 2018, 172(6):1216-1227.

26. Tabah A, Koulenti D, Laupland K, Misset B, Valles J, Bruzzi de Carvalho F, Paiva JA, Cakar N, Ma X, Eggimann P et al: Characteristics and determinants of outcome of hospitalacquired bloodstream infections in intensive care units: the EUROBACT International Cohort Study. Intensive Care Med 2012, 38(12):1930-1945.

27. Raymond F, Boissinot M, Ouameur AA, Deraspe M, Plante PL, Kpanou SR, Berube E, Huletsky A, Roy PH, Ouellette M et al: Culture-enriched human gut microbiomes reveal core and accessory resistance genes. Microbiome 2019, 7(1):56.

28. Walker GT, Quan J, Higgins SG, Toraskar N, Chang W, Saeed A, Sapiro V, Pitzer K, Whitfield N, Lopansri BK et al: Predicting Antibiotic Resistance in Gram-Negative Bacilli from Resistance Genes. Antimicrob Agents Chemother 2019, 63(4). 
29. Livanos AE, Snider EJ, Whittier S, Chong DH, Wang TC, Abrams JA, Freedberg DE: Rapid gastrointestinal loss of Clostridial Clusters IV and XIVa in the ICU associates with an expansion of gut pathogens. PLoS One 2018, 13(8):e0200322.

30. Terry MA, Freedberg DE, Morris MC: An Alternative Consent Process for Minimal Risk Research in the ICU. Crit Care Med 2017, 45(9):1450-1456.

31. Budding AE, Grasman ME, Eck A, Bogaards JA, Vandenbroucke-Grauls CM, van Bodegraven AA, Savelkoul PH: Rectal swabs for analysis of the intestinal microbiota. PLoS One 2014, 9(7):e101344.

32. Singer M, Deutschman CS, Seymour CW, Shankar-Hari M, Annane D, Bauer M, Bellomo R, Bernard GR, Chiche JD, Coopersmith CM et al: The Third International Consensus Definitions for Sepsis and Septic Shock (Sepsis-3). JAMA 2016, 315(8):801-810.

33. Baggs J, Fridkin SK, Pollack LA, Srinivasan A, Jernigan JA: Estimating National Trends in Inpatient Antibiotic Use Among US Hospitals From 2006 to 2012. JAMA Intern Med 2016, 176(11):1639-1648.

34. (CLSI) CaLSI. Performance standards for antimicrobial susceptibility testing. CLSI Supplement M100. 2017(27th Ed.).

35. Fosbrink M, Wilt G, Chen L, Kreisworth B, Devgan V: Identification of antibiotic resistance genes in Klebsiella pneumoniae isolates and metagenomic samples using realtime PCR arrays. Sample to Insight, accessed on-line at https://wwwslidesharenet/QIAGENscience/identification-of-antibiotic-resistance-genesin-klebsiella-pneumoniae-isolates-and-metagenomic-samples-using-realtime-pcr-arrays April 6, 2016.

36. Rocha DJ, Santos CS, Pacheco LG: Bacterial reference genes for gene expression studies by RT-qPCR: survey and analysis. Antonie Van Leeuwenhoek 2015, 108(3):685-693.

37. Freedberg DE, Salmasian H, Cohen B, Abrams JA, Larson EL: Receipt of Antibiotics in Hospitalized Patients and Risk for Clostridium difficile Infection in Subsequent Patients Who Occupy the Same Bed. JAMA Intern Med 2016, 176(12):1801-1808. 
Table 1. Baseline characteristics of the patients in the study, treatments received in the $4 \quad$ ICU, and clinical outcomes within 30 days.

\begin{tabular}{ll}
\hline Baseline characteristic & $\mathbf{N}(\boldsymbol{\%})$ \\
& $\mathbf{N}$ total=48 \\
\hline Age (median years, IQR) & $64(52-74)$ \\
Female & $21(44 \%)$ \\
Admitted to ICU from hospital floor & $12(25 \%)$ \\
Baseline immunosuppression & $18(38 \%)$ \\
Primary reason for ICU admission, organized by organ system & \\
$\quad$ Cardiovascular/shock & $14(29 \%)$ \\
$\quad$ Respiratory failure & $10(21 \%)$ \\
$\quad$ Neurological & $7(15 \%)$ \\
$\quad$ Gastrointestinal & $6(13 \%)$ \\
$\quad$ Liver & $5(10 \%)$ \\
$\quad$ Malignancy & $3(6 \%)$ \\
Renal failure & $3(6 \%)$ \\
\hline
\end{tabular}

Treatments received in the ICU, from the time of admission until 72 hours later Antibiotics

Any antibiotics

$41(85 \%)$

Broad-spectrum antibiotics

$39(81 \%)$

Non-antibiotic interventions

Enteral feeding $\quad 36(75 \%)$

Opioids

$35(73 \%)$

Mechanical ventilation

$26(54 \%)$

Proton pump inhibitors

$22(46 \%)$

Hemodialysis

$6(13 \%)$

\begin{tabular}{ll}
\hline Clinical outcomes within 30 days of ICU admission* & \\
\hline Culture-proven infections & $19(40 \%)$ \\
MDR infections & $14(29 \%)$ \\
Death & $11(23 \%)$
\end{tabular}

Immunosuppression was defined as history of solid organ transplant or as receipt of ablative chemotherapy, steroids at the equivalent of $\geq 5 \mathrm{mg}$ /day prednisone, antimetabolites, anti-TNF $\alpha$ agents, calcineurin inhibitors, or mycophenolate. Broadspectrum antibiotics were $\beta$-lactam/ $\beta$-lactamase inhibitor combination antibiotics, cephalosporins, fluoroquinolones, lincosamides (clindamycin), and monobactams (e.g., meropenem). *See reference [37] for operationalization of culture-proven infections; MDR infections were the subset of culture-proven infections caused by MRSA, VRE, and Gram-negative bacteria with non-susceptibility to $3^{\text {rd }}$ generation cephalosporins. 
2 Supplemental Table 1. Antibiotic resistance genes tested with qPCR, organized by category.

\begin{tabular}{|c|c|}
\hline Category & Genes \\
\hline Aminoglycoside & aacC1, aacC2, aacC4, aadA1, apha6 \\
\hline Class A beta-lactamase & $\begin{array}{l}\text { BES-1, BIC-1, CTX-M-1, CTX-M-8, CTX-M-9, GES, IMI \& } \\
\text { NMC-A, KPC, Per-1, Per-2, SFC-1, SFO-1, SHV (156D) } \\
\text { (156G) (238G240E) (238G240K) (238S240E) (238S240K), } \\
\text { SME, TLA-1, VEB }\end{array}$ \\
\hline Class B beta-lactamase & $\begin{array}{l}\text { ccrA, IMP-1, IMP-12, IMP-2, IMP-5, NDM, VIM-1, VIM-13, } \\
\text { VIM-7 }\end{array}$ \\
\hline Class C beta-lactamase & $\begin{array}{l}\text { ACC-1, ACC-3, ACT 5/7, ACT-1, CFE-1, CMY-10, DHA, } \\
\text { FOX, LAT, MIR, MOX }\end{array}$ \\
\hline Class D beta-lactamase & $\begin{array}{l}\text { OXA-10, OXA-18, OXA-2, OXA-23, OXA-24, OXA-45, } \\
\text { OXA-48, OXA-50, OXA-51, OXA-54, OXA-55, OXA-58, } \\
\text { OXA-60 }\end{array}$ \\
\hline Erythromycin & ereB \\
\hline Fluoroquinolone & $\begin{array}{l}\text { AAC(6)-Ib-cr, QepA, QnrA, QnrB-1, QnrB-31, QnrB-4, } \\
\text { QnrB-5, QnrB-8, QnrC, QnrD, QnrS }\end{array}$ \\
\hline $\begin{array}{l}\text { Macrolide- } \\
\text { Lincosamide }\end{array}$ & ermA, ermB, ermC, mefA, msrA \\
\hline MDR efflux pump & oprj, oprm \\
\hline Tetracycline & tet $\mathrm{A}$, tetB \\
\hline Beta-lactam & mecA \\
\hline Vancomycin & vanB, vanC \\
\hline PVL chain $F$ & lukF \\
\hline IgG binding protein $\mathrm{A}$ & spa \\
\hline
\end{tabular}


1 Supplemental Table 2. Sequential Organ Failure Assessment (SOFA) 2 characteristics and scores, calculated at the time of ICU admission and 72 3 hours later.

\begin{tabular}{|c|c|c|}
\hline SOFA characteristics & $\begin{array}{l}\text { ICU admission } \\
\mathbf{N}(\%)\end{array}$ & $\begin{array}{l}72 \text { hours } \\
\mathbf{N}(\%)\end{array}$ \\
\hline $\begin{array}{l}\text { Respiration: Ventilator support at the } \\
\text { time of assessment }\end{array}$ & $26(54 \%)$ & $14(29 \%)$ \\
\hline $\begin{array}{l}\text { Respiration: } \mathrm{PaO}_{2} / \mathrm{FiO}_{2}<400 \text { or }<200 \\
\text { with ventilator support }\end{array}$ & $20(42 \%)$ & $9(19 \%)$ \\
\hline $\begin{array}{l}\text { Coagulation: platelet count }<150 \mathrm{x} \\
10^{3} / \mathrm{mm}^{3}\end{array}$ & $28(58 \%)$ & $25(52 \%)$ \\
\hline Liver: serum bilirubin $>1.2 \mathrm{mg} / \mathrm{dL}$ & $11(23 \%)$ & $14(29 \%)$ \\
\hline $\begin{array}{c}\text { Shock: mean arterial pressure }<70 \\
\text { mm Hg or use of vasopressors }\end{array}$ & $22(46 \%)$ & $19(40 \%)$ \\
\hline $\begin{array}{l}\text { Neurologic: Glasgow coma scale }<15 \\
\text { points* }\end{array}$ & $26(54 \%)$ & $19(40 \%)$ \\
\hline $\begin{array}{l}\text { Renal: Serum creatinine }>1.2 \mathrm{mg} / \mathrm{dL} \\
\text { or urine output }<500 \mathrm{~mL} / \mathrm{day}^{* *}\end{array}$ & $24(50 \%)$ & $28(58 \%)$ \\
\hline $\begin{array}{l}\text { Total SOFA points } \\
\text { (median, IQR) }\end{array}$ & $16(10-18)$ & $17(15-19)$ \\
\hline
\end{tabular}

4 SOFA criteria were calculated based on the worst values during the preceding 524 hours. *Glasgow coma scale (GCS) was the assumed GCS absent sedating 6 medications. **SOFA urine output criteria was not applied for patients with 7 baseline end-stage renal disease $(n=2)$. 
3 Figure 1. Receipt of antibiotics during the study. (A) Distribution of the number of patients

4 receiving each antibiotic during the 72 hours of the study. (B) Heatmap of pairwise antibiotic

5 combinations. The numbers on the heatmap denote the number of patients receiving each drug

6 pair during the 72 hours of the study.

7

8 Figure 2. Change in antimicrobial resistance phenotype stratified by receipt of antibiotics. The 9 distribution of cultures showing non-susceptibility after 72 hours in the ICU is shown for (A) 10 Gram-negative bacteria with $\beta$-lactam resistance, (B) vancomycin-resistant Enterococcus (VRE) 11 and (C) methicillin-resistant Staphylococcus aureus (MRSA). This is shown after 72 hours in the 12 ICU for individuals that tested negative at admission. P-values are for Fisher's test, comparing 13 resistance after 72 hours based on receipt of antibiotics within the relevant category.

Figure 3. Within-individual changes in antimicrobial resistance genotype stratified by receipt of 16 antibiotics. Genotypic changes are based on the difference in $\mathrm{C}_{\mathrm{T}}$ values from admission to after 1772 hours (i.e., admission $\mathrm{C}_{\mathrm{T}}$ value minus 72 hour $\mathrm{C}_{\mathrm{T}}$ value for each gene). The within-individual 18 changes $\left(\mathrm{dC}_{\mathrm{T}}\right)$ are displayed as raw data with an overlay of box-and-whisker plots for individual 19 and combined genes conferring resistance against $\beta$-lactams, vancomycin, and macrolides. In all 20 of the antibiotic class-based categories, antibiotics were not associated with changes in 21 antimicrobial resistance genotype. 
1 Figure 4. Relationship between individuals' resistance pattern at ICU admission compared to 72

2 hours later, for antimicrobial resistance phenotype and genotype. (A) Heatmap depicting the

3 percentage of patients with resistant isolates at 72 hours that also had resistant isolates at

4 admission. Results were filtered to only include antibiotics for which resistance was present in at

5 least 10 samples. (B) Heatmap depicting the percentage of patients with resistance genes at 72

6 hours that also had resistance genes at admission. Again, results have been filtered to only show

7 genes for which resistance was present in at least 10 samples. For both heatmaps, horizontal axes

8 are resistance at admission and vertical axes are resistance after 72 hours. Bottom rows show (1)

9 relative risk (RR) of testing positive for a resistance category at 72 hours for those that were

10 resistant to the same category at admission versus those who tested negative on admission; and (2)

$11 \mathrm{p}$-values for the same comparison. In panel $\mathrm{B}$, the cases of $\mathrm{RR}=0$ reflect that no sample-pairs

12 were positive on both admission and 72 hours later.

14 Supplemental Figure 1. Incidence of antimicrobial resistance phenotype and genotype at ICU 15 admission and 72 hours later. (A) Incidence of resistance phenotype based on culture for $\beta$-lactam 16 resistance in Gram-negative bacteria, MRSA, and VRE. There was no significant increase in 17 resistance after 72 hours although there were trends in that direction. (B) Incidence of resistance 18 genotype based on qPCR. The resistance genes are grouped by the antibiotic class they confer 19 resistance to. Samples were considered positive for a given gene if the $\mathrm{C}_{\mathrm{T}}$ value was $\leq 34$, There 20 was no significant increase in resistance genotype after 72 hours. Chi-squared or Fisher's p-values 21 are shown. 
A

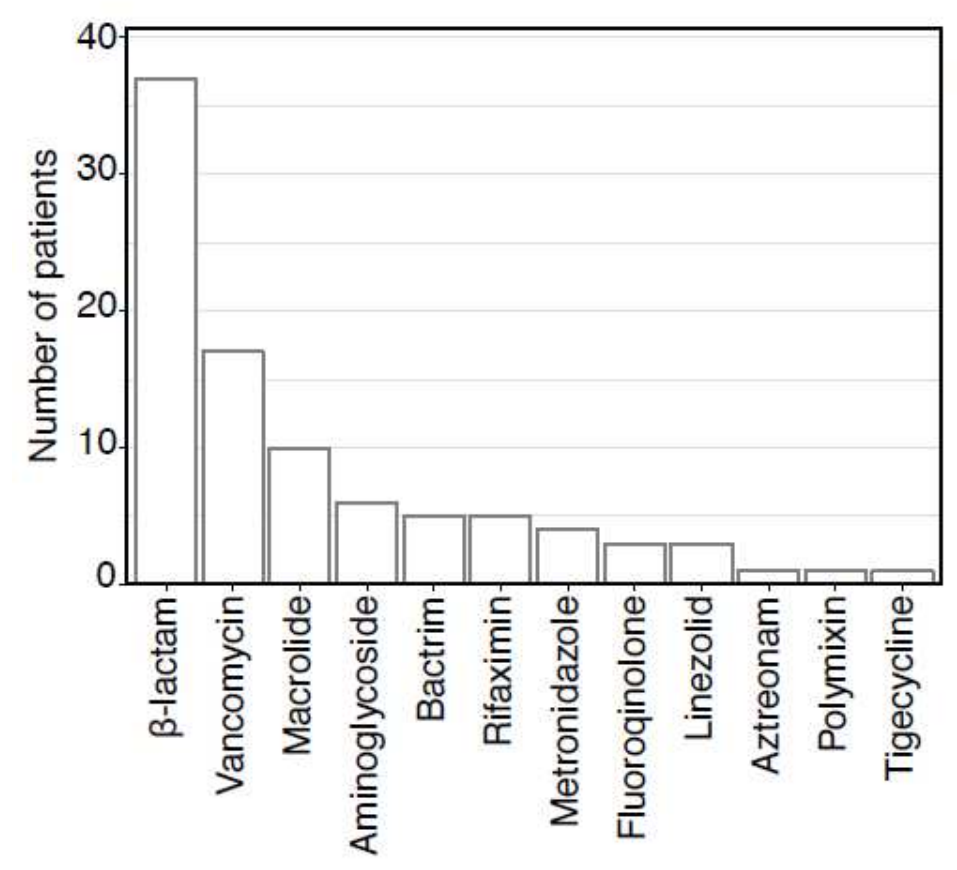

B $\frac{\text { क्ष }}{00}$

Aminoglycoside

Aztreonam

Bactrim

$\beta$-lactam

Fluoroqinolone

Linezolid

Macrolide

Metronidazole

Polymixin

\begin{tabular}{l|l|l|}
\cline { 3 - 3 } Rifaximin & 2 & 1 \\
\hline
\end{tabular}

Tigecycline

Vancomycin

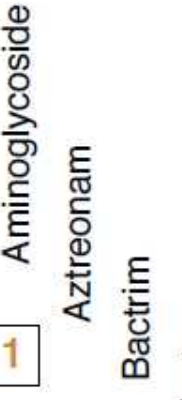

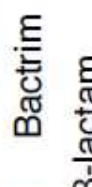

के

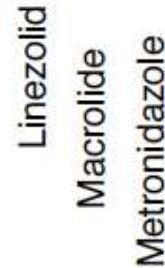

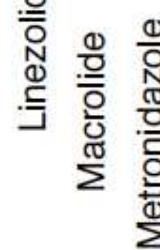

2

\section{Figure 1}

Receipt of antibiotics during the study. (A) Distribution of the number of patients receiving each antibiotic during the 72 hours of the study. (B) Heatmap of pairwise antibiotic combinations. The numbers on the heatmap denote the number of patients receiving each drug pair during the 72 hours of the study.

\section{Gram negative}

$\beta$-lactam resistance

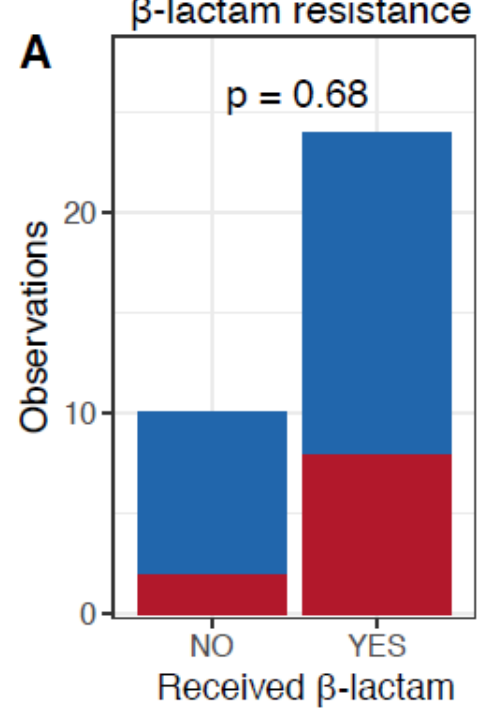

VRE

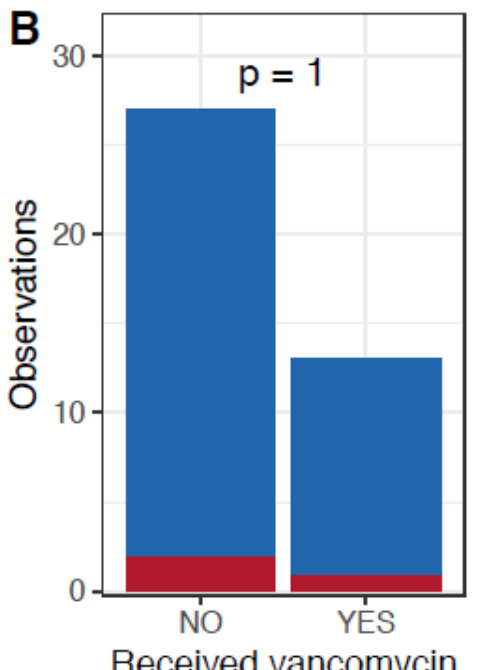

MRSA

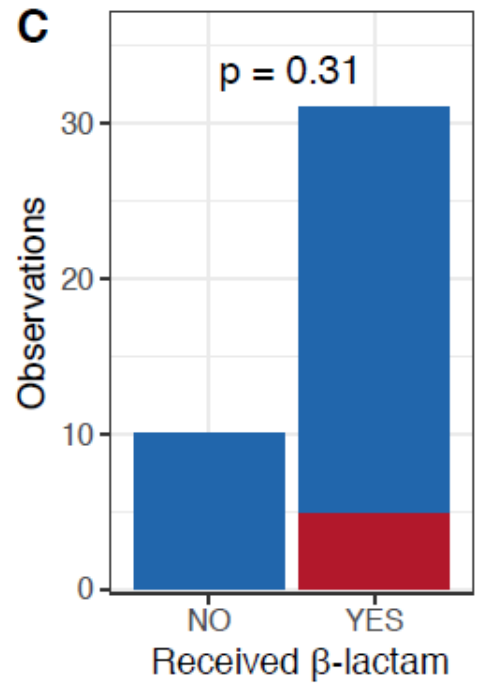

Resistance after 72 hours

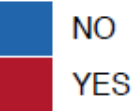


Change in antimicrobial resistance phenotype stratified by receipt of antibiotics. The distribution of cultures showing non-susceptibility after 72 hours in the ICU is shown for (A) Gram-negative bacteria with $\beta$-lactam resistance, (B) vancomycin-resistant Enterococcus (VRE) and (C) methicillin-resistant Staphylococcus aureus (MRSA). This is shown after 72 hours in the ICU for individuals that tested negative at admission. P-values are for Fisher's test, comparing resistance after 72 hours based on receipt of antibiotics within the relevant category.

A

安

:

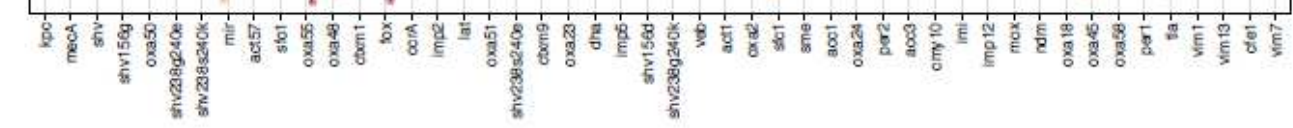

c

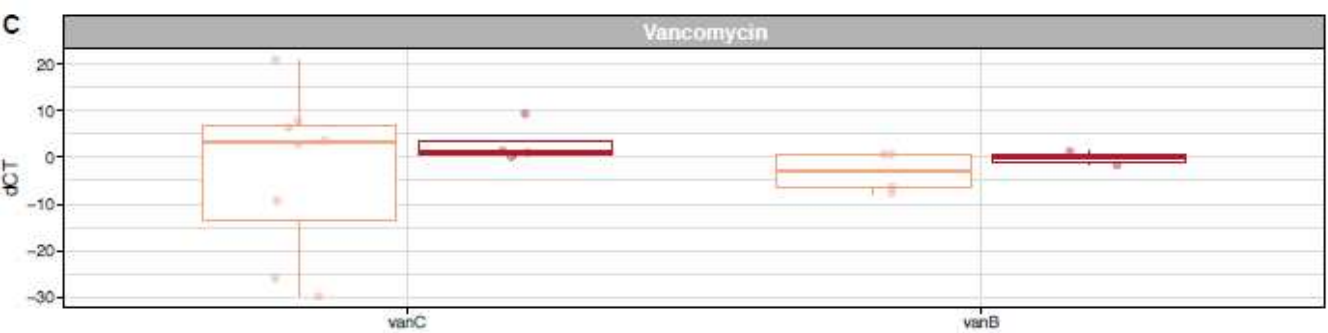

E

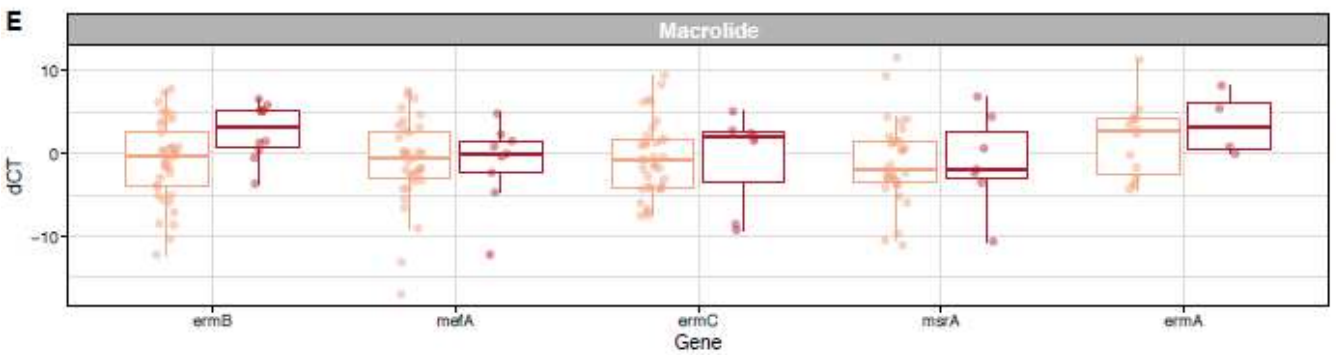

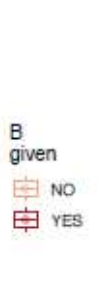

B

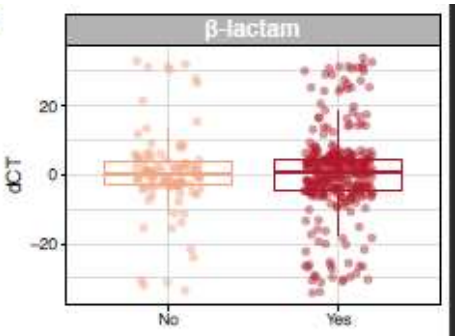

D
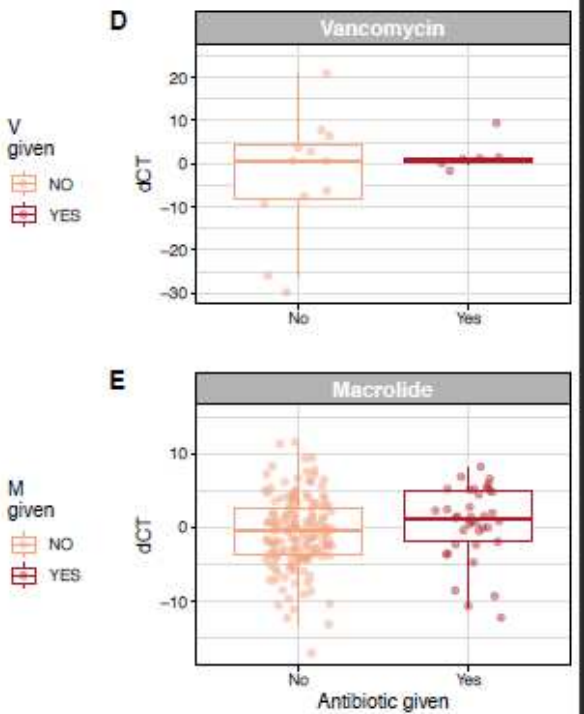

E

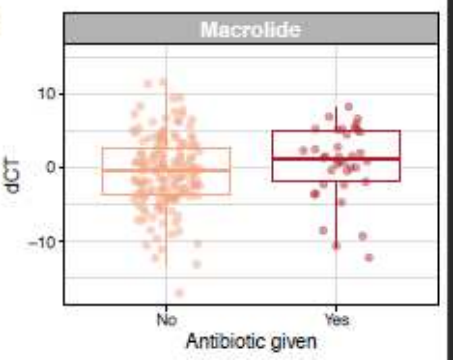

Figure 3

Within-individual changes in antimicrobial resistance genotype stratified by receipt of antibiotics. Genotypic changes are based on the difference in CT values from admission to after 72 hours (i.e., admission CT value minus 72 hour CT value for each gene). The within-individual changes (dCT) are displayed as raw data with an overlay of box-and-whisker plots for individual and combined genes conferring resistance against $\beta$-lactams, vancomycin, and macrolides. In all of the antibiotic class-based categories, antibiotics were not associated with changes in antimicrobial resistance genotype. 


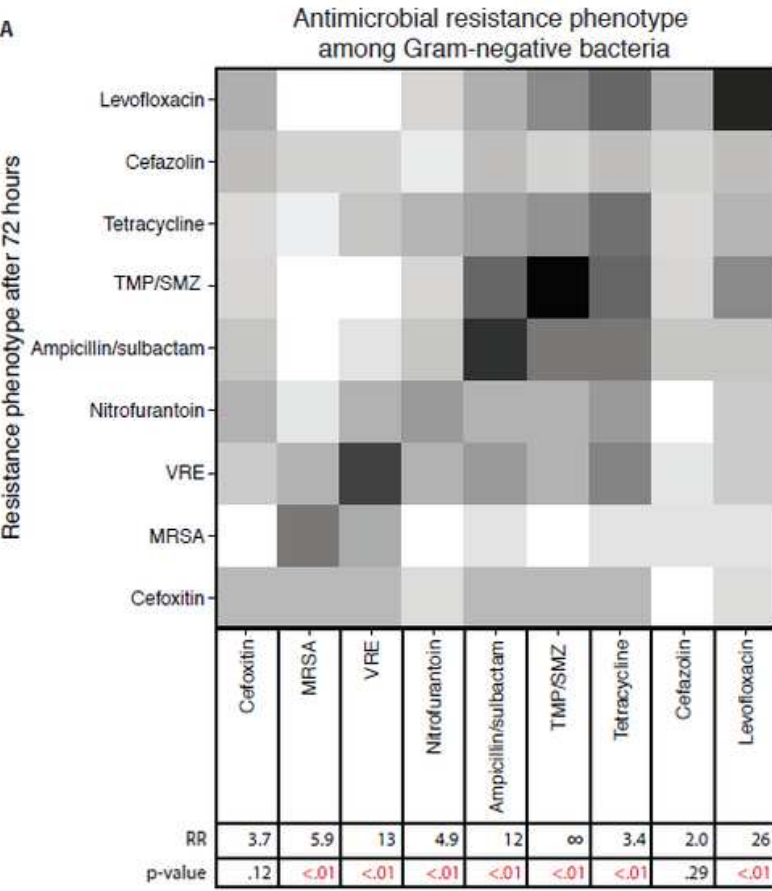

Resistance phenotype at ICU admission

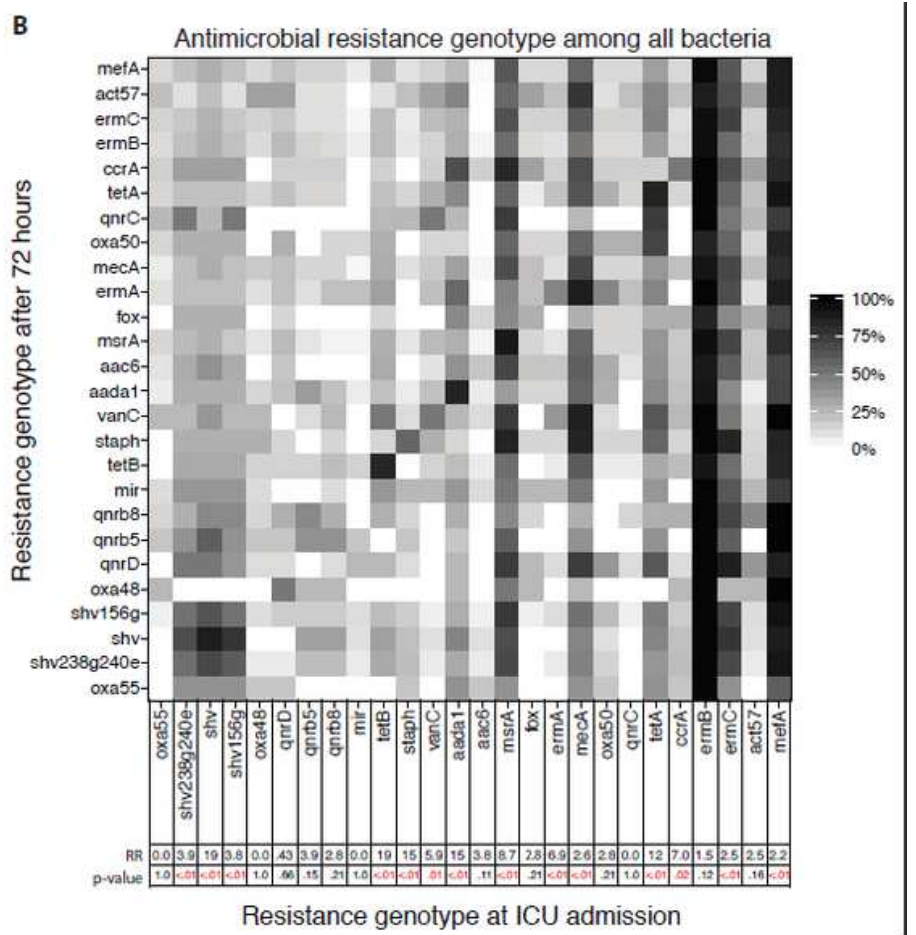

\section{Figure 4}

Relationship between individuals' resistance pattern at ICU admission compared to 72 hours later, for antimicrobial resistance phenotype and genotype. (A) Heatmap depicting the percentage of patients with resistant isolates at 72 hours that also had resistant isolates at admission. Results were filtered to only include antibiotics for which resistance was present in at least 10 samples. (B) Heatmap depicting the percentage of patients with resistance genes at 72 hours that also had resistance genes at admission. Again, results have been filtered to only show genes for which resistance was present in at least 10 samples. For both heatmaps, horizontal axes are resistance at admission and vertical axes are resistance after 72 hours. Bottom rows show (1) relative risk (RR) of testing positive for a resistance category at 72 hours for those that were resistant to the same category at admission versus those who tested negative on admission; and (2) p-values for the same comparison. In panel B, the cases of $\mathrm{RR}=0$ reflect that no sample-pairs were positive on both admission and 72 hours later.

\section{Supplementary Files}

This is a list of supplementary files associated with this preprint. Click to download.

- FigureS1.pdf

- Datasupplement1.xlsx

- Datasupplement2.xIsx 